\begin{tabular}{|c|c|c|}
\hline & Int.J.Curr.Microbiol.App.Sci (2021) 10(12): 391-403 & \\
\hline & $\begin{array}{l}\text { International Journal of Current Microbiology and Applied Sciences } \\
\text { ISSN: 2319-7706 Volume } \mathbf{1 0} \text { Number } \mathbf{1 2} \mathbf{( 2 0 2 1 )} \\
\text { Journal homepage: } \underline{\text { http://www.ijcmas.com }}\end{array}$ & 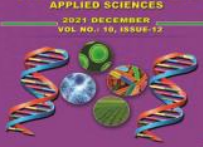 \\
\hline $\begin{array}{l}\text { EXCELLENT } \\
\text { PUBLISHERS }\end{array}$ & & \\
\hline
\end{tabular}

\title{
The Involvement of Grandparents in Bringing up Grandchildren and their Views about it
}

\author{
Jaya Bangale* and Smita Patil
}
Department of Human Development and Family Studies, College of Community Science, Vasantrao Naik Marathwada Krishi Vidyapeeth, Parbhani - 431 402, MS, India

*Corresponding author

\begin{tabular}{|l|}
\hline Ke y w o r d s \\
$\begin{array}{l}\text { Grandparents, } \\
\text { grandchildren, } \\
\text { upbringing, views, } \\
\text { involvement }\end{array}$ \\
\hline Article Info \\
\hline $\begin{array}{l}\text { Received: } \\
\text { 10 November } 2021 \\
\text { Accepted: } \\
\text { 06 December } 2021 \\
\text { Available Online: } \\
\text { 10 December } 2021\end{array}$ \\
\hline \hline
\end{tabular}

\section{Introduction}

In India since ancient times, the families are tied up with unseen bond, cooperation, harmony, interdependence and also they are the most important child care institute. Children are expected to grow under the glory of family where a satisfactory upbringing of children is ensured. Family members mother, father, siblings and grandparents play a very crucial role in child's wholesome development. The unconditional warmth and infinite love towards each other create a special bond between them. Over the past few 
decades with changing family patterns, increased life expectancy, growing number of dual- workers households and higher rates of family breakdown, grandparents are now playing a prominent role in their grandchildren's lives.

Many research studies had indicated that children who live with their grandparents become more matured from a psychological point of view because they receive value education from them and are happier also as every child needs a dependable, consistent and positive relationship with at least one adult in order to achieve overall optimal development and health. Pong and Chen (2010) found that the children who live together with their grandparents do better on their academic tasks. Similarly, Barnett et al., (2010) observed that grandparents' involvement reduce grandchildren's negative emotions and enhance their social competence. Hamilton (2005) in his research study found that having a grandparent in the household was associated with lower depressive symptoms and less deviant behaviour in children. A study carried out by sonuga- Barke and Mistry (2000) found that in a sample of Muslim and Hindu families, children exhibited better behavioural adjustment when grandparents were involved in their care than when they were not involved. Recent research conducted by the University of Oxford highlights the important role of grandparents in ensuring children's well-being. The report authored by Prof. Ann Buchanan of the department of social policy and intervention, says that a high level of grandparental involvement increases the wellbeing of children. A study of more than 1,500 children showed that those with a high level of grandparental involvement had fewer emotional and behavioural problems. Viceversa, grandchildren have a positive influence on grandparents. A 2013 study by Boston College researchers Sara Moorman found that a close, emotional relationship between grandparents and grandchildren can have a measurable effect on the psychological wellbeing of both grandparents and grandchildren.

Therefore by considering above facts it is felt necessary to study the involvement of grandparents in bringing up grandchildren and their views about it.

\section{Materials and Methods}

A sample of 150 children (5-7 yrs old), 80 belonging to middle SES group and 70 belonging to low SES group and brought up by their both parents (either both parents are working or only one parent is working) as well as by their grandparents were chosen randomly from the selected 10 colonies from Parbhani City of Marathwada region of Maharashtra state. The data pertaining to the study were collected by personally interviewing the grandparents of sample children based on structured and open ended interview schedule and also by administering Kuppuswamy's Socio-economic Status Scale revised by Dr. Sheikh Mohd Saleem (2018) for assessing the socio-economic status of the sample children. Further while categorizing the sample children, the children brought up by grandparents and both employed parents were considered in Group 1 while Children brought up by grandparents and employed fathers and non employed mothers were considered in Group 2.The data thus collected were pooled, tabulated, statistically analyzed and discussed.

\section{Results and Discussion}

The personal background information of the sample children is indicated in table 1 . Irrespective of their socioeconomic status and the groups of children, relatively a higher percentage of them were in the age group of 5 - 6 yrs (50-54\%) and remaining of them were in the age group of $6-7$ yrs. (45-49\%). 
With respect to their ordinal position, it was noticed that a higher percentage of them were first born (55 - 58\%) followed by middle born $(38 \%)$ and last born (3-5\%). Forty six to fifty per cent of the sample children were male, while the remaining of the sample children were female irrespective of socio-economic status and groups of children. Further 51 - 54 per cent of the sample children were recorded to be studying in I std. followed by in Sr. KG $(25-33 \%)$ and in Jr. KG (14 - 20\%). On the whole, irrespective of the socio-economic status and groups of children, it was observed that relatively a higher percentage of them were in the age group of $5-6$ yrs and studying in I std.

Table 2 indicates background information of the grandparents of the sample children. In group 1, irrespective of the socio-economic status, a majority of the grandmothers were found to be in the age group of 55 to $65 \mathrm{yrs}$ (37 - 43\%) followed by 60 to 70 yrs (37 $39 \%), 45$ to 55 yrs $(8-13 \%)$ and above 70 yrs old $(10 \%)$. Whereas 46 - 53 per cent grandfathers' age was between 46 yrs and 53 yrs followed by in the range of 55 - 65 yrs (25 - 40\%), 65 - 70 yrs (13 - 17\%) and 45 - 55 yrs (4\%). In group 2, with regard to the grandmothers' age, irrespective of the socioeconomic status, similar trend was observed among them, while 27 - 45 per cent grandfathers were in the age group of 65 to 70 yrs followed by above 70 yrs (21 - 37\%), 55 to 65 yrs (30 - 35\%) and 45 to 55 yrs old (18\%). As far as grandparents' education was concerned, irrespective of the groups and socio-economic status, it was observed that majority of the grandmothers were non literate (24 - 52\%) followed by secondary school educated (13 - 41\%), primary school educated (6 - 27\%), high school educated (6-13\%), middle school educated (4 - 10\%), graduates $(2-8 \%)$ and postgraduates $(3-4 \%)$. Whereas, majority of the grandfathers were recorded to be graduates $(16-45 \%)$, middle school educated (6 - 40\%), higher secondary school educated $(8-32 \%)$, nonliterates $(6-24 \%)$, postgraduates $(2-21 \%)$, primary school educated $(3-18 \%)$ and secondary school educated $(6-14 \%)$.

Table 3 illustrates information about the grandparents involved in child upbringing. In group 1, irrespective of the socio-economic status, relatively a higher percentage of both the paternal grandparents were found to be involved in upbringing of sample children (65\%). Besides this 20 per cent paternal grandmothers and 5 per cent grandfathers also were recorded to be involved in the upbringing of their grandchildren. In group 2, almost similar trend was recorded with regard to the paternal grandparents' involvement in upbringing of their grandchildren. On the other hand as far as maternal grandparents' involvement in upbringing of sample children was concerned, it was noticed that irrespective of the socio-economic status in group 1, 7 per cent both the grandparents and a meagre percentage of grandmothers were involved in it. Whereas in group 2, irrespective of the socio-economic status, 6 per cent both grandparents, 7 per cent only grandmothers and a meagre percentage of grandfathers' involvement $(1 \%)$ was observed in upbringing of their grandchildren. Further irrespective of the socio-economic status a very higher percentage of the grandparents $(94 \%)$ reported that right from the neonatal stage of sample children, they were involved in their upbringing. Whereas about 5 per cent grandparents' involvement in upbringing of their grandchildren was observed to be only for specific months of the years.

Significantly a higher percentage of both the middle SES grandparents of group 1 were observed to be involved in upbringing of children as compared to their counterparts in group 2. Whereas significantly more number of middle SES grandmothers in group 2 were 
found to have involved in upbringing of children. On the whole, it is obvious from the results that irrespective of the groups and socio-economic status of the sample children, a higher percentage of both the paternal grandparents were found to be involved in child upbringing right from their neonatal stage.

Grandparental feedbacks towards their involvement in upbringing of grandchildren are depicted in table 4 and fig. 1.1. In group 1, irrespective of the socio-economic status all the grandparents expressed that, it was most rewarding experience of their life and their grandchildren were a very precious gift which they got in their life (92\%). About 80-98 per cent of them opined that they felt happy and contented for their contribution in grandchildren's upbringing (98\%), spent time joyfully with each other (96\%), got purpose to lead a satisfying life (89\%) and privileged to got opportunity to guide and groom young generation $(83 \%)$ and because of grandchildren's presence in home, they hardly felt lonely $(80 \%)$. The corresponding percentages in group 2 were found to be 100 , 97, 93, 97, 77, 91, 77 and 66. In addition to these, irrespective of the groups and socioeconomic status of the families 33 - 43 per cent of the grandparents opined that, their involvement in grandchildren's upbringing was beneficial to them for remaining physically and mentally fit as well as also for refining and modernizing them to lead their life comfortably. Based on the groups, statistically non significant differences were observed among them with regard to their views about their involvement in the upbringing of grandchildren. The findings are in support of some of the results observed by Hodgson et al., (1992), Wearing B(1996), Mills et al., (2001), Shinde and Patnam
(2003), Backhouse and Graham (2013) and Jaya Bangale (2014). Drawbacks reported by the grandparents due to their involvement in upbringing of grandchildren are detailed in table 5 and fig.1.2. In group 1, irrespective of the socio-economic status, relatively a higher percentage of the grandparents $(71 \%)$ reported that due to their involvement in upbringing of grandchildren, sometimes it became difficult for them to carry out their personal work like performing religious activities, reading books/ newspapers, chitchatting with neighbours etc.

Whereas 40 - 58 per cent of them stated that due to their involvement in upbringing of grandchildren they could not take nap, joined programmes which were arranged by their friends and relatives (47\%), and sometimes even could not view interesting serials on television. About 38 per cent grandparents expressed that their grandchildren used to disoblige them in bad moods. Besides these about 35 per cent were found to have discrepancies between them and their children (parents) about perceptions of child upbringing practices sometimes which lead to conflicts among them. The corresponding percentages in group 2 were 44.21, 28.42, 10.52, 30.52, and 33.68. Few significant differences were noted in the grandparent's opinions about the drawbacks of their involvement in the upbringing of their grandchildren.

Irrespective of the groups and socio-economic status of the sample children, a higher percentage of both the paternal grandparents were found to be involved in child upbringing right from their neonatal stage. With regard to the involvement in upbringing of grandchildren all the grandparents expressed that, it was most rewarding experience of their life. 
Table.1 Personal background information of the sample children

\begin{tabular}{|c|c|c|c|c|c|c|}
\hline \multirow{4}{*}{$\begin{array}{l}\text { Background } \\
\text { Information }\end{array}$} & \multicolumn{6}{|c|}{ SES and percentages of children } \\
\hline & \multicolumn{3}{|c|}{ Group 1} & \multicolumn{3}{|c|}{ Group 2} \\
\hline & $\begin{array}{l}\text { MSES } \\
(n=30)\end{array}$ & $\begin{array}{c}\text { LSES } \\
(n=25)\end{array}$ & $\begin{array}{l}\text { Irrespective of } \\
\text { SES }(n=55)\end{array}$ & $\begin{array}{l}\text { MSES } \\
(n=50)\end{array}$ & $\begin{array}{c}\text { LSES } \\
(n=45)\end{array}$ & $\begin{array}{c}\text { Irrespecti ve } \\
\text { of SES }(n=95)\end{array}$ \\
\hline & $\mathbf{a}$ & b & c & d & $\mathbf{e}$ & f \\
\hline \multicolumn{7}{|c|}{ Chronological Age (yrs) } \\
\hline $5-6$ & $\begin{array}{c}56.66 \\
(17)\end{array}$ & $\begin{array}{c}52.00 \\
(13)\end{array}$ & $\begin{array}{c}54.54 \\
(30)\end{array}$ & $\begin{array}{c}52.00 \\
(26)\end{array}$ & $\begin{array}{c}48.88 \\
(22)\end{array}$ & $\begin{array}{c}50.52 \\
(48)\end{array}$ \\
\hline $6-7$ & $\begin{array}{c}43.33 \\
(13)\end{array}$ & $\begin{array}{l}48.00 \\
(12)\end{array}$ & $\begin{array}{c}45.45 \\
(25)\end{array}$ & $\begin{array}{l}48.00 \\
(24)\end{array}$ & $\begin{array}{c}51.11 \\
(23)\end{array}$ & $\begin{array}{c}49.47 \\
(47)\end{array}$ \\
\hline \multicolumn{7}{|c|}{ Ordinal Position } \\
\hline First born & $\begin{array}{c}60.00 \\
(18)\end{array}$ & $\begin{array}{l}56.00 \\
(14)\end{array}$ & $\begin{array}{c}58.18 \\
(32)\end{array}$ & $\begin{array}{c}60.00 \\
(30)\end{array}$ & $\begin{array}{c}51.11 \\
(23)\end{array}$ & $\begin{array}{c}55.78 \\
(53)\end{array}$ \\
\hline Middle born & $\begin{array}{c}36.66 \\
(11)\end{array}$ & $\begin{array}{l}40.00 \\
(10)\end{array}$ & $\begin{array}{l}38.18 \\
(21)\end{array}$ & $\begin{array}{c}38.00 \\
(19)\end{array}$ & $\begin{array}{c}40.00 \\
(18)\end{array}$ & $\begin{array}{c}38.94 \\
(37)\end{array}$ \\
\hline Last born & $\begin{array}{l}3.33 \\
(1)\end{array}$ & $\begin{array}{c}4.00 \\
(1)\end{array}$ & $\begin{array}{l}03.63 \\
(2)\end{array}$ & $\begin{array}{c}2.00 \\
(1)\end{array}$ & $\begin{array}{c}8.88 \\
(4)\end{array}$ & $\begin{array}{c}05.26 \\
(5)\end{array}$ \\
\hline \multicolumn{7}{|c|}{ Gender } \\
\hline Male & $\begin{array}{c}43.33 \\
(13)\end{array}$ & $\begin{array}{c}60.00 \\
(15)\end{array}$ & $\begin{array}{l}50.90 \\
(28)\end{array}$ & $\begin{array}{l}40.00 \\
(20)\end{array}$ & $\begin{array}{c}53.33 \\
(24)\end{array}$ & $\begin{array}{c}46.31 \\
(44)\end{array}$ \\
\hline Female & $\begin{array}{l}56.66 \\
(17)\end{array}$ & $\begin{array}{l}40.00 \\
(10)\end{array}$ & $\begin{array}{c}49.09 \\
(27)\end{array}$ & $\begin{array}{c}60.00 \\
(30)\end{array}$ & $\begin{array}{c}46.66 \\
(21)\end{array}$ & $\begin{array}{c}53.68 \\
(51)\end{array}$ \\
\hline \multicolumn{7}{|c|}{ Standard } \\
\hline Jr. KG & $\begin{array}{c}23.33 \\
(7)\end{array}$ & $\begin{array}{l}28.00 \\
(4)\end{array}$ & $\begin{array}{c}20.00 \\
(11)\end{array}$ & $\begin{array}{l}14.00 \\
(7)\end{array}$ & $\begin{array}{c}15.55 \\
(7)\end{array}$ & $\begin{array}{c}14.73 \\
(14)\end{array}$ \\
\hline Sr. KG & $\begin{array}{c}26.66 \\
(8)\end{array}$ & $\begin{array}{c}24.00 \\
(6)\end{array}$ & $\begin{array}{c}25.45 \\
(14)\end{array}$ & $\begin{array}{c}34.00 \\
(17)\end{array}$ & $\begin{array}{c}33.33 \\
(15)\end{array}$ & $\begin{array}{c}33.68 \\
(32)\end{array}$ \\
\hline 1 std. & $\begin{array}{c}50.00 \\
(15)\end{array}$ & $\begin{array}{c}60.00 \\
(15)\end{array}$ & $\begin{array}{c}54.54 \\
(30) \\
\end{array}$ & $\begin{array}{c}52.00 \\
(26)\end{array}$ & $\begin{array}{c}51.11 \\
(23)\end{array}$ & $\begin{array}{c}51.57 \\
(49)\end{array}$ \\
\hline
\end{tabular}

Figures in parenthesis indicate frequencies

Group 1 - Children brought up by grandparents and both employed parents

Group 2 - Children brought up by grandparents, employed fathers and non employed mothers or only employed mothers 
Table.2 Background information of the grandparents of the sample children

\begin{tabular}{|c|c|c|c|c|c|c|c|c|}
\hline \multirow{5}{*}{$\begin{array}{l}\text { Background } \\
\text { Information }\end{array}$} & \multicolumn{8}{|c|}{ SES and percentages of grandparents } \\
\hline & \multicolumn{4}{|c|}{ Group 1} & \multicolumn{4}{|c|}{ Group 2} \\
\hline & \multicolumn{2}{|c|}{ MSES } & \multicolumn{2}{|c|}{ LSES } & \multirow{2}{*}{\multicolumn{2}{|c|}{$\begin{array}{c}\text { MSES } \\
\text { c }\end{array}$}} & \multirow{2}{*}{\multicolumn{2}{|c|}{$\begin{array}{c}\text { LSES } \\
\text { d }\end{array}$}} \\
\hline & \multicolumn{2}{|c|}{$\mathbf{a}$} & \multicolumn{2}{|c|}{ b } & & & & \\
\hline & $\begin{array}{c}\mathbf{G M} \\
(\mathbf{n}=29)\end{array}$ & $\begin{array}{c}\text { GF } \\
(\mathbf{n}=\mathbf{2 8})\end{array}$ & $\begin{array}{c}\text { GM } \\
(\mathbf{n}=\mathbf{2 3})\end{array}$ & $\underset{(\mathbf{n}=15)}{\mathbf{G F}}$ & $\begin{array}{c}\text { GM } \\
(\mathbf{n}=\mathbf{4 8})\end{array}$ & $\underset{(\mathbf{n}=33)}{\mathbf{G F}}$ & $\begin{array}{c}\text { GM } \\
(\mathrm{n}=\mathbf{4 4})\end{array}$ & $\underset{(\mathbf{n}=37}{\mathbf{G F}}$ \\
\hline \multicolumn{9}{|l|}{ Age (yrs) } \\
\hline $45-55$ & $\begin{array}{l}13.79 \\
(4)\end{array}$ & $\begin{array}{l}03.57 \\
(1)\end{array}$ & $\begin{array}{c}08.69 \\
(2)\end{array}$ & - & $\begin{array}{c}10.41 \\
(5)\end{array}$ & $\begin{array}{l}03.03 \\
(1)\end{array}$ & $\begin{array}{c}18.18 \\
(8)\end{array}$ & - \\
\hline $55-65$ & $\begin{array}{c}37.93 \\
(11)\end{array}$ & $\begin{array}{c}25.00 \\
(7)\end{array}$ & $\begin{array}{c}43.47 \\
(10)\end{array}$ & $\begin{array}{c}40.00 \\
(6)\end{array}$ & $\begin{array}{c}45.83 \\
(22)\end{array}$ & $\begin{array}{c}30.30 \\
(10)\end{array}$ & $\begin{array}{c}45.45 \\
(20)\end{array}$ & $\begin{array}{c}35.13 \\
(13)\end{array}$ \\
\hline $65-70$ & $\begin{array}{c}37.93 \\
(11)\end{array}$ & $\begin{array}{c}17.85 \\
(5)\end{array}$ & $\begin{array}{c}39.13 \\
(9)\end{array}$ & $\begin{array}{c}13.33 \\
(2)\end{array}$ & $\begin{array}{c}41.66 \\
(20)\end{array}$ & $\begin{array}{c}45.45 \\
(15)\end{array}$ & $\begin{array}{c}34.09 \\
(15)\end{array}$ & $\begin{array}{c}27.02 \\
(10)\end{array}$ \\
\hline Above 70 & $\begin{array}{c}10.34 \\
(3)\end{array}$ & $\begin{array}{c}53.57 \\
(15)\end{array}$ & $\begin{array}{c}08.69 \\
(2)\end{array}$ & $\begin{array}{c}46.66 \\
(7)\end{array}$ & $\begin{array}{c}02.08 \\
(1)\end{array}$ & $\begin{array}{c}21.21 \\
(7)\end{array}$ & $\begin{array}{l}02.27 \\
(1)\end{array}$ & $\begin{array}{c}37.83 \\
(14)\end{array}$ \\
\hline \multicolumn{9}{|l|}{ Education } \\
\hline Non literates & $\begin{array}{c}24.13 \\
(7)\end{array}$ & $\begin{array}{l}10.71 \\
(3)\end{array}$ & $\begin{array}{c}26.08 \\
(6)\end{array}$ & $\begin{array}{l}06.66 \\
(1)\end{array}$ & $\begin{array}{c}35.41 \\
(17)\end{array}$ & $\begin{array}{l}06.06 \\
(2)\end{array}$ & $\begin{array}{l}52.27 \\
(23)\end{array}$ & $\begin{array}{c}24.32 \\
(9)\end{array}$ \\
\hline $\begin{array}{c}\text { Primary } \\
\text { school } \\
\text { educated }\end{array}$ & $\begin{array}{l}06.80 \\
(2)\end{array}$ & $\begin{array}{l}03.57 \\
(1)\end{array}$ & $\begin{array}{c}17.39 \\
(4)\end{array}$ & $\begin{array}{l}06.66 \\
(1)\end{array}$ & $\begin{array}{c}22.91 \\
(11)\end{array}$ & $\begin{array}{l}12.12 \\
(4)\end{array}$ & $\begin{array}{l}27.27 \\
(12)\end{array}$ & $\begin{array}{c}18.91 \\
(7)\end{array}$ \\
\hline $\begin{array}{l}\text { Middle school } \\
\text { educated }\end{array}$ & $\begin{array}{c}10.34 \\
(3)\end{array}$ & - & $\begin{array}{c}04.34 \\
(1)\end{array}$ & $\begin{array}{c}40.00 \\
(6)\end{array}$ & $\begin{array}{c}10.41 \\
(5)\end{array}$ & $\begin{array}{c}06.06 \\
(2)\end{array}$ & $\begin{array}{c}04.54 \\
(2)\end{array}$ & $\begin{array}{c}18.91 \\
(7)\end{array}$ \\
\hline SSC & $\begin{array}{c}41.37 \\
(12)\end{array}$ & $\begin{array}{c}14.28 \\
(4)\end{array}$ & $\begin{array}{c}30.43 \\
(7)\end{array}$ & $\begin{array}{c}06.66 \\
(1)\end{array}$ & $\begin{array}{c}18.75 \\
(9)\end{array}$ & $\begin{array}{c}12.12 \\
(4)\end{array}$ & $\begin{array}{c}13.63 \\
(6)\end{array}$ & $\begin{array}{c}10.81 \\
(4)\end{array}$ \\
\hline HSC & $\begin{array}{c}06.80 \\
(2)\end{array}$ & $\begin{array}{c}32.14 \\
(9)\end{array}$ & $\begin{array}{c}13.04 \\
(3)\end{array}$ & $\begin{array}{c}20.00 \\
(3)\end{array}$ & $\begin{array}{c}06.25 \\
(3)\end{array}$ & $\begin{array}{c}12.12 \\
(4)\end{array}$ & - & $\begin{array}{c}\mathbf{0 8 . 1 0} \\
(3)\end{array}$ \\
\hline Graduates & $\begin{array}{c}06.80 \\
(2)\end{array}$ & $\begin{array}{c}17.85 \\
(5)\end{array}$ & $\begin{array}{c}08.69 \\
(2)\end{array}$ & $\begin{array}{c}20.00 \\
(3)\end{array}$ & $\begin{array}{c}04.16 \\
(2)\end{array}$ & $\begin{array}{c}45.45 \\
(15)\end{array}$ & $\begin{array}{l}02.27 \\
(1)\end{array}$ & $\begin{array}{c}16.21 \\
(6)\end{array}$ \\
\hline $\begin{array}{l}\text { Postgraduates } \\
\text { / Doctorates }\end{array}$ & $\begin{array}{c}03.44 \\
\text { (1) }\end{array}$ & $\begin{array}{c}21.42 \\
(6)\end{array}$ & - & - & $\begin{array}{c}04.16 \\
(2)\end{array}$ & $\begin{array}{c}06.06 \\
(2)\end{array}$ & - & $\begin{array}{c}02.70 \\
(1)\end{array}$ \\
\hline
\end{tabular}

Figures in parenthesis indicate frequencies

Group 1 - Children brought up by grandparents and both employed parents

Group 2 - Children brought up by grandparents, employed fathers and non employed mothers or only employed mother

GM - Grandmothers, GF - Grandfathers 
Table.3 Information about the grandparents involved in upbringing of sample children

\begin{tabular}{|c|c|c|c|c|c|c|c|c|c|}
\hline \multirow{4}{*}{$\begin{array}{c}\text { Grandparents involved in child } \\
\text { upbringing }\end{array}$} & \multicolumn{6}{|c|}{ SES and percentages of grandparents } & \multicolumn{3}{|c|}{ Z Values } \\
\hline & \multicolumn{3}{|c|}{ Group 1} & \multicolumn{3}{|c|}{ Group 2} & \multirow{3}{*}{ a Vs d } & \multirow{3}{*}{ b Vs e } & \multirow{3}{*}{ c Vs f } \\
\hline & $\begin{array}{l}\text { MSES } \\
(\mathrm{n}=30)\end{array}$ & $\begin{array}{c}\text { LSES } \\
(n=25)\end{array}$ & $\begin{array}{c}\text { Irrespective } \\
\text { of SES } \\
(n=55)\end{array}$ & $\begin{array}{l}\text { MSES } \\
(\mathbf{n}=\mathbf{5 0})\end{array}$ & $\begin{array}{c}\text { LSES } \\
(n=45)\end{array}$ & $\begin{array}{c}\text { Irrespective } \\
\text { of SES } \\
(n=95)\end{array}$ & & & \\
\hline & $\mathbf{a}$ & $\mathbf{b}$ & c & d & $\mathbf{e}$ & $\mathbf{f}$ & & & \\
\hline \multicolumn{10}{|l|}{ Paternal } \\
\hline Both grandparents & $\begin{array}{l}80.00 \\
(24)\end{array}$ & $\begin{array}{l}48.00 \\
(12)\end{array}$ & $\begin{array}{c}65.45 \\
(36)\end{array}$ & $\begin{array}{l}52.00 \\
(26)\end{array}$ & $\begin{array}{c}77.77 \\
(35)\end{array}$ & $\begin{array}{c}64.21 \\
(61)\end{array}$ & $2.75 * *$ & $0.48^{\mathrm{NS}}$ & $0.15^{\mathrm{NS}}$ \\
\hline Grandmothers & $\begin{array}{c}03.33 \\
(1)\end{array}$ & $\begin{array}{l}40.00 \\
(10)\end{array}$ & $\begin{array}{l}20.00 \\
(11)\end{array}$ & $\begin{array}{c}26.00 \\
(13)\end{array}$ & $\begin{array}{c}11.11 \\
(5)\end{array}$ & $\begin{array}{c}18.94 \\
(18)\end{array}$ & $3.23 * *$ & $0.40^{\mathrm{NS}}$ & $0.15^{\mathrm{NS}}$ \\
\hline Grandfathers & $\begin{array}{c}03.33 \\
(1)\end{array}$ & $\begin{array}{l}08.00 \\
(2)\end{array}$ & $\begin{array}{l}05.45 \\
(3)\end{array}$ & $\begin{array}{l}02.00 \\
(1)\end{array}$ & $\begin{array}{l}02.22 \\
(1)\end{array}$ & $\begin{array}{l}02.10 \\
(2)\end{array}$ & $0.34^{\mathrm{NS}}$ & $0.08^{\mathrm{NS}}$ & $0.98^{\mathrm{NS}}$ \\
\hline \multicolumn{10}{|l|}{ Maternal } \\
\hline Both grandparents & $\begin{array}{c}10.00 \\
(3)\end{array}$ & $\begin{array}{l}04.00 \\
(1)\end{array}$ & $\begin{array}{l}07.27 \\
(4)\end{array}$ & $\begin{array}{c}10.00 \\
(5)\end{array}$ & $\begin{array}{l}02.22 \\
(1)\end{array}$ & $\begin{array}{c}06.31 \\
(6)\end{array}$ & - & $0.04^{\mathrm{NS}}$ & $0.39^{\mathrm{NS}}$ \\
\hline Grandmothers & $\begin{array}{c}03.33 \\
(1)\end{array}$ & - & $\begin{array}{l}01.81 \\
(1)\end{array}$ & $\begin{array}{l}08.00 \\
(4)\end{array}$ & $\begin{array}{l}06.66 \\
(3)\end{array}$ & $\begin{array}{c}07.36 \\
(7)\end{array}$ & $0.92^{\mathrm{NS}}$ & - & - \\
\hline Grandfathers & - & - & - & $\begin{array}{l}02.00 \\
(1)\end{array}$ & - & $\begin{array}{l}01.05 \\
(1)\end{array}$ & - & - & - \\
\hline \multicolumn{10}{|l|}{ Duration of grand parenting } \\
\hline $\begin{array}{c}\text { Right from neonatal stage of sample } \\
\text { children }\end{array}$ & $\begin{array}{l}96.66 \\
(29)\end{array}$ & $\begin{array}{l}92.00 \\
(23)\end{array}$ & $\begin{array}{c}94.54 \\
(52)\end{array}$ & $\begin{array}{l}92.00 \\
(46)\end{array}$ & $\begin{array}{c}97.77 \\
(44)\end{array}$ & $\begin{array}{c}94.73 \\
(90)\end{array}$ & $0.92^{\mathrm{NS}}$ & $0.92^{\mathrm{NS}}$ & $0.98^{\mathrm{NS}}$ \\
\hline For specific months or years & $\begin{array}{c}03.33 \\
(1)\end{array}$ & $\begin{array}{c}08.00 \\
(2)\end{array}$ & $\begin{array}{c}05.45 \\
(3)\end{array}$ & $\begin{array}{c}08.00 \\
(4)\end{array}$ & $\begin{array}{c}02.22 \\
(1)\end{array}$ & $\begin{array}{c}05.26 \\
(5)\end{array}$ & $0.92^{\mathrm{NS}}$ & $0.08^{N S}$ & $0.98^{\mathrm{NS}}$ \\
\hline
\end{tabular}


Table.4 Grandparental views towards their involvement in upbringing of grandchildren

\begin{tabular}{|c|c|c|c|c|c|c|c|c|c|}
\hline \multirow{4}{*}{ Grandparental Feedback } & \multicolumn{6}{|c|}{ SES and percentages of children } & \multirow{2}{*}{\multicolumn{3}{|c|}{ Z Values }} \\
\hline & \multicolumn{3}{|c|}{ Group 1} & \multicolumn{3}{|c|}{ Group 2} & & & \\
\hline & $\begin{array}{l}\text { MSES } \\
(\mathbf{n}=30)\end{array}$ & $\begin{array}{l}\text { LSES } \\
(n=25)\end{array}$ & $\begin{array}{c}\text { Irrespe } \\
\text { ctive } \\
\text { of SES } \\
(n=55)\end{array}$ & $\begin{array}{l}\text { MSES } \\
(\mathbf{n}=\mathbf{5 0})\end{array}$ & $\begin{array}{c}\text { LSES } \\
(n=45)\end{array}$ & $\begin{array}{l}\text { Irrespective } \\
\text { of SES } \\
(n=95)\end{array}$ & a Vs c & b Vs d & c Vsf \\
\hline & $\mathbf{a}$ & b & c & d & $\mathbf{e}$ & $\mathbf{f}$ & & & \\
\hline Most rewarding experience of life & $\begin{array}{c}100.00 \\
(30)\end{array}$ & $\begin{array}{c}100.00 \\
(25)\end{array}$ & $\begin{array}{c}100.00 \\
(55)\end{array}$ & $\begin{array}{c}100.00 \\
(50)\end{array}$ & $\begin{array}{c}100.00 \\
(45)\end{array}$ & $100.00(95)$ & - & - & - \\
\hline $\begin{array}{l}\text { Feel happy and contented for } \\
\text { contributing in } \\
\text { grandchildren's upbringing }\end{array}$ & $\begin{array}{c}100.00 \\
(30)\end{array}$ & $\begin{array}{l}96.00 \\
(24)\end{array}$ & $\begin{array}{l}98.18 \\
(54)\end{array}$ & $\begin{array}{l}100.00 \\
(50)\end{array}$ & $\begin{array}{c}93.33 \\
(42)\end{array}$ & $96.84(92)$ & - & $0.49^{\mathrm{NS}}$ & $0.65^{\mathrm{NS}}$ \\
\hline $\begin{array}{l}\text { Spend time with each other } \\
\text { joyfully }\end{array}$ & $\begin{array}{l}100.00 \\
(30)\end{array}$ & $\begin{array}{l}92.00 \\
(23)\end{array}$ & $\begin{array}{c}96.36 \\
(53)\end{array}$ & $\begin{array}{c}98.00 \\
(49)\end{array}$ & $\begin{array}{c}97.77 \\
(44)\end{array}$ & 97.89 (93) & $1.39^{\mathrm{NS}}$ & $0.98^{\mathrm{NS}}$ & $0.52^{\mathrm{NS}}$ \\
\hline $\begin{array}{c}\text { Grandchild is a very precious gift } \\
\text { in life }\end{array}$ & $\begin{array}{l}93.33 \\
(28)\end{array}$ & $\begin{array}{l}92.00 \\
(23)\end{array}$ & $\begin{array}{c}92.72 \\
(51)\end{array}$ & $\begin{array}{c}90.00 \\
(45)\end{array}$ & $\begin{array}{c}97.77 \\
(44)\end{array}$ & $93.68(89)$ & $0.53^{\mathrm{NS}}$ & $0.98^{\mathrm{NS}}$ & $0.22^{\mathrm{NS}}$ \\
\hline Got purpose to lead a satisfying life & $\begin{array}{l}93.33 \\
(28)\end{array}$ & $\begin{array}{c}84.00 \\
(21)\end{array}$ & $\begin{array}{c}89.09 \\
(49)\end{array}$ & $\begin{array}{c}86.00 \\
(43)\end{array}$ & $\begin{array}{c}77.77 \\
(35)\end{array}$ & $82.10(78)$ & $1.09^{\mathrm{NS}}$ & $0.64^{\mathrm{NS}}$ & $1.21^{\mathrm{NS}}$ \\
\hline $\begin{array}{c}\text { Privilege to guide and groom } \\
\text { young } \\
\text { generation }\end{array}$ & $\begin{array}{c}93.33 \\
(28)\end{array}$ & $\begin{array}{c}72.00 \\
(18)\end{array}$ & $\begin{array}{c}83.63 \\
(46)\end{array}$ & $\begin{array}{c}90.00 \\
(45)\end{array}$ & $\begin{array}{c}84.44 \\
(38)\end{array}$ & $87.36(83)$ & $0.53^{\mathrm{NS}}$ & $1.18^{\mathrm{NS}}$ & $0.61^{\mathrm{NS}}$ \\
\hline
\end{tabular}


Table.4 Contd...

\begin{tabular}{|c|c|c|c|c|c|c|c|c|c|}
\hline \multirow{3}{*}{ Grandparental Feedback } & \multicolumn{3}{|c|}{ Group 1} & \multicolumn{3}{|c|}{ Group 2} & \multicolumn{3}{|c|}{$Z$ value } \\
\hline & $\begin{array}{l}\text { MSES } \\
(n=30)\end{array}$ & $\begin{array}{c}\text { LSES } \\
(n=25)\end{array}$ & $\begin{array}{c}\text { Irrespe } \\
\text { ctive } \\
\text { of SES } \\
(n=55)\end{array}$ & $\begin{array}{l}\text { MSES } \\
(n=50)\end{array}$ & $\begin{array}{l}\text { LSES } \\
(n=45)\end{array}$ & $\begin{array}{c}\text { Irrespective } \\
\text { of SES } \\
(n=95)\end{array}$ & \multirow[t]{2}{*}{ a Vs c } & \multirow[t]{2}{*}{ b Vs d } & \multirow[t]{2}{*}{ c Vs f } \\
\hline & $\mathbf{a}$ & b & $\mathbf{C}$ & d & $\mathbf{e}$ & $\mathbf{f}$ & & & \\
\hline Helps in recalling parenting practices & $\begin{array}{l}90.00 \\
(27)\end{array}$ & $\begin{array}{l}84.00 \\
(21)\end{array}$ & $\begin{array}{c}87.27 \\
(48)\end{array}$ & $\begin{array}{l}92.00 \\
(46)\end{array}$ & $\begin{array}{c}91.11 \\
(41)\end{array}$ & $91.57(87)$ & $0.29^{\mathrm{NS}}$ & $0.83^{\mathrm{NS}}$ & $0.80^{\mathrm{NS}}$ \\
\hline Hardly feel lonely & $\begin{array}{l}83.33 \\
(25)\end{array}$ & $\begin{array}{l}76.00 \\
(19)\end{array}$ & $\begin{array}{c}80.00 \\
(44)\end{array}$ & $\begin{array}{l}70.00 \\
(35)\end{array}$ & $\begin{array}{c}66.66 \\
(30)\end{array}$ & $68.42(65)$ & $1.41^{\mathrm{NS}}$ & $0.84^{\mathrm{NS}}$ & $1.60^{\mathrm{NS}}$ \\
\hline $\begin{array}{l}\text { Help to remain physically and } \\
\text { mentally fit }\end{array}$ & $\begin{array}{l}46.66 \\
(14)\end{array}$ & $\begin{array}{c}40.00 \\
(10)\end{array}$ & $\begin{array}{c}43.63 \\
(24)\end{array}$ & $\begin{array}{l}34.00 \\
(17)\end{array}$ & $\begin{array}{c}33.33 \\
(15)\end{array}$ & $33.68(32)$ & $1.11^{\mathrm{NS}}$ & $0.55^{\mathrm{NS}}$ & $1.20^{\mathrm{NS}}$ \\
\hline Help in refining and modernizing self & $\begin{array}{c}46.66 \\
(14)\end{array}$ & $\begin{array}{c}32.00 \\
(8)\end{array}$ & $\begin{array}{c}40.00 \\
(22)\end{array}$ & $\begin{array}{l}36.00 \\
(18)\end{array}$ & $\begin{array}{l}31.11 \\
(14)\end{array}$ & $33.68(32)$ & $0.93^{\mathrm{NS}}$ & $0.07^{\mathrm{NS}}$ & $0.77^{\mathrm{NS}}$ \\
\hline
\end{tabular}

Figures in parenthesis indicate frequencies

$* \mathbf{P}<0.05$ level

$* * \mathbf{P}<0.01$ level

NS - Non-significant

Group 1 - Children brought up by grandparents and both employed parents

Group 2 - Children brought up by grandparents, employed fathers and non employed mothers or only employed mother 
Table.5 Drawbacks reported by the grandparents due to their involvement in upbringing of grandchildren

\begin{tabular}{|c|c|c|c|c|c|c|c|c|c|}
\hline \multirow{4}{*}{ Drawbacks } & \multicolumn{6}{|c|}{ SES and percentages of children } & \multirow{2}{*}{\multicolumn{3}{|c|}{ Z Values }} \\
\hline & \multicolumn{3}{|c|}{ Group 1} & \multicolumn{3}{|c|}{ Group 2} & & & \\
\hline & $\begin{array}{l}\text { MSES } \\
(n=30)\end{array}$ & $\begin{array}{c}\text { LSES } \\
(\mathrm{n}=25)\end{array}$ & $\begin{array}{c}\text { Irrespective } \\
\text { of SES } \\
(n=55)\end{array}$ & $\begin{array}{l}\text { MSES } \\
(n=50)\end{array}$ & $\begin{array}{c}\text { LSES } \\
(n=45)\end{array}$ & $\begin{array}{c}\text { Irrespective } \\
\text { of SES } \\
(n=95)\end{array}$ & a vs c & b vs d & c vs f \\
\hline & $\mathbf{a}$ & b & c & d & $\mathbf{e}$ & $\mathbf{f}$ & & & \\
\hline $\begin{array}{c}\text { Sometimes could not do } \\
\text { personal } \\
\text { work }\end{array}$ & $\begin{array}{c}70.00 \\
(21)\end{array}$ & $\begin{array}{c}72.00 \\
(18)\end{array}$ & $\begin{array}{c}70.90 \\
(39)\end{array}$ & $\begin{array}{c}48.00 \\
(24)\end{array}$ & $\begin{array}{c}40.00 \\
(18)\end{array}$ & $\begin{array}{c}44.21 \\
(42)\end{array}$ & $2.00 *$ & $2.76^{* *}$ & $3.35 * *$ \\
\hline Affects nap time & $\begin{array}{c}60.00 \\
(18)\end{array}$ & $\begin{array}{l}56.00 \\
(14)\end{array}$ & $\begin{array}{c}58.18 \\
(32)\end{array}$ & $\begin{array}{c}30.00 \\
(15)\end{array}$ & $\begin{array}{c}26.66 \\
(12)\end{array}$ & $\begin{array}{c}28.42 \\
(27)\end{array}$ & $2.71 * *$ & $2.46^{*}$ & $3.67 * *$ \\
\hline $\begin{array}{l}\text { Could not join programmes of } \\
\text { friends and relatives }\end{array}$ & $\begin{array}{c}50.00 \\
(15)\end{array}$ & $\begin{array}{c}44.00 \\
(11)\end{array}$ & $\begin{array}{c}47.27 \\
(26)\end{array}$ & $\begin{array}{c}20.00 \\
(10)\end{array}$ & -- & $\begin{array}{c}10.52 \\
(10)\end{array}$ & $2.79 * *$ & -- & $4.94 * *$ \\
\hline $\begin{array}{l}\text { Sometimes could not view } \\
\text { interesting TV serials }\end{array}$ & $\begin{array}{c}43.33 \\
(13)\end{array}$ & $\begin{array}{l}36.00 \\
(9)\end{array}$ & $\begin{array}{c}40.00 \\
(22)\end{array}$ & $\begin{array}{c}32.00 \\
(16)\end{array}$ & $\begin{array}{c}28.88 \\
(13)\end{array}$ & $\begin{array}{c}30.52 \\
(29)\end{array}$ & $1.01^{\mathrm{NS}}$ & $0.60^{\mathrm{NS}}$ & $1.16^{\mathrm{NS}}$ \\
\hline $\begin{array}{l}\text { Discrepancies between them } \\
\text { and their children (parents) } \\
\text { about perceptions of child } \\
\text { upbringing } \\
\text { practices }\end{array}$ & $\begin{array}{c}36.66 \\
(11)\end{array}$ & $\begin{array}{c}32.00 \\
(8)\end{array}$ & $\begin{array}{c}34.54 \\
(19)\end{array}$ & $\begin{array}{c}32.00 \\
(16)\end{array}$ & $\begin{array}{c}35.55 \\
(16)\end{array}$ & $\begin{array}{c}33.68 \\
(32)\end{array}$ & $0.42^{\mathrm{NS}}$ & $0.30^{\mathrm{NS}}$ & $0.10^{\mathrm{NS}}$ \\
\hline $\begin{array}{c}\text { Disoblige of grandchildren } \\
\text { due to } \\
\text { bad moods }\end{array}$ & $\begin{array}{c}33.33 \\
(10)\end{array}$ & $\begin{array}{c}44.00 \\
(11)\end{array}$ & $\begin{array}{c}38.18 \\
(21)\end{array}$ & $\begin{array}{c}30.00 \\
(15)\end{array}$ & $\begin{array}{c}35.55 \\
(16)\end{array}$ & $\begin{array}{c}32.63 \\
(31)\end{array}$ & $0.30^{\mathrm{NS}}$ & $0.69^{\mathrm{NS}}$ & $0.68^{\mathrm{NS}}$ \\
\hline
\end{tabular}

Figures in parenthesis indicate frequencies

$* \mathbf{P}<\mathbf{0 . 0 5}$ level $* * \mathbf{P}<\mathbf{0 . 0 1}$ level $\mathbf{N S}$ - Non-significant

Group 1 - Children brought up by grandparents and both employed parents

Group 2 - Children brought up by grandparents, employed fathers and non employed mothers or only employed mother 
Fig.1
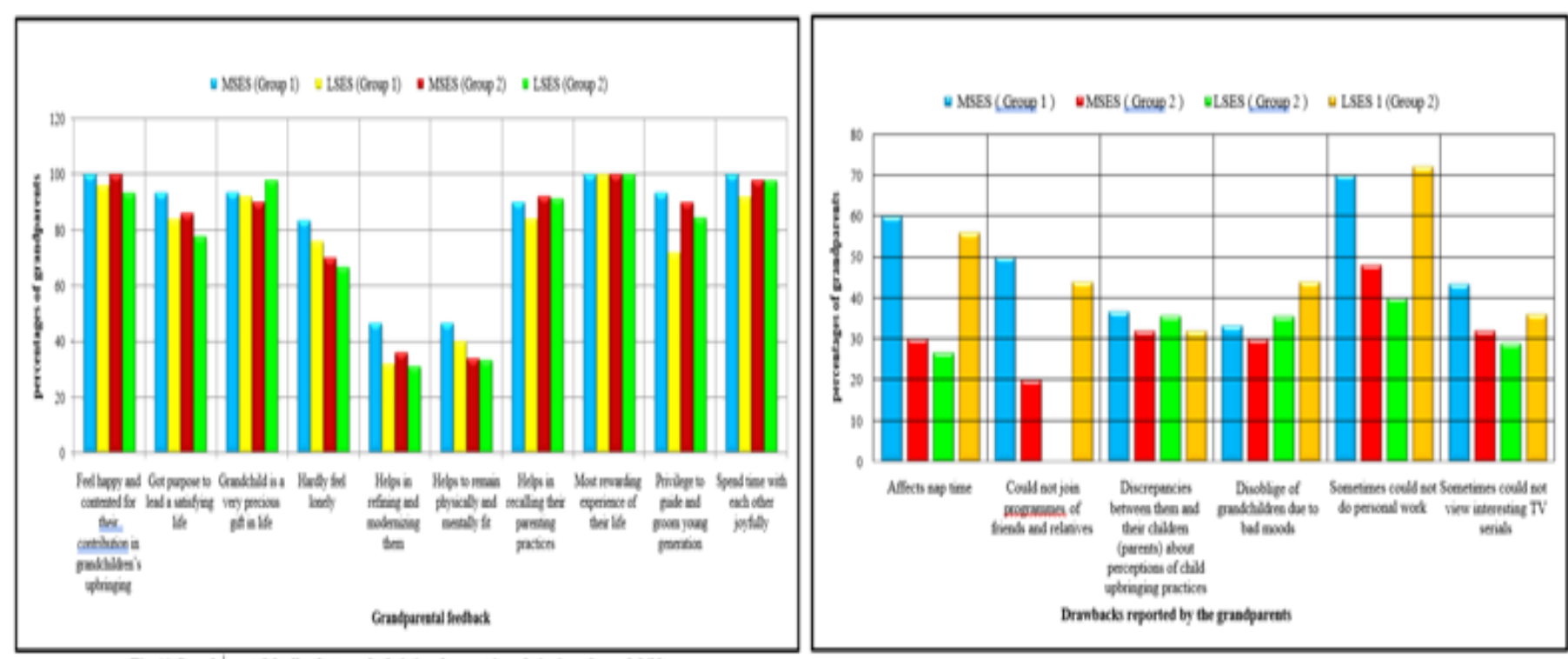

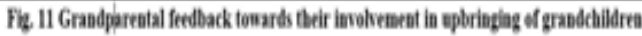

Fig 12 Drantacks reported by the grandpareats due to their iavolvemeat in upbringiag of grasdchildrea 
In addition to it about 66-98 per cent of them opined that they felt happy and contented for their contribution in grandchildren's upbringing, spent time joyfully with each other (96-97\%), got purpose to lead a satisfying life (82-89\%) and privileged to got opportunity to guide and groom young generation (84-87\%) and because of grandchildren's presence in home, they hardly felt lonely (68-80\%) and also it was beneficial to them for remaining physically and mentally fit $(31-43 \%)$. However, they also expressed that sometimes it became difficult for them to carry out their personal work due to their involvement in upbringing of grandchildren (44- $71 \%$ ). On the whole, the findings of the study highlight that families need to involve grandparents in caring grandchildren as they are found to be playing vital role in child upbringing and which in turn enabling grandparents to lead happy, contented and dignified life.

\section{Acknowledgement}

The authors are thankful to the grandparents and to their families for providing required data to carry out this research work. The authors also acknowledge concerned authorities of VNMKV, Parbhani for providing essential facilities to conduct this research study.

\section{References}

Backhouse, Jan and Graham, Anne (2013) Grandparents Raising Their grandchildren: Acknowledging the Experience of Grief, Australian Social work :66(3) 440-454.

Bangale Jaya (2014). A study on involvement of urban grandparents in grandchild care and development. RCC Report, VNMKV, Parbhani

Barnett, M. A., Scaramella, L. V., Neppl, T. K., Ontai, L. L., \& Conger, R. D.
(2010). Grandmother involvement as a protective factor for early childhood social adjustment. Journal of Family psychology, 24(5), 635- 645. https://doi.org/10.1037/a0020829

Buchanan Ann Grandparents contribute to children's wellbeing, University of Oxford

https://www.ox.ac.uk/research/researc h-impact/grandparents-contributechildrens- wellbeing

Hamilton, H. (2005). Extended families and adolescent well-being. Journal of Adolescent Health. 36(3), 260-266

Hodgson, E., and Lynne, G. (1992). Adult grandchildren and their grandparents: Their enduring bond. International $\mathrm{J}$ of Aging and Human Development 34(3) 209-225

Kathleen sullivan (2013) Study Boosts Grandparent-Grandchildren Ties. BC News, https://www.bc.edu/bcweb/resources/agora-portal-r.html

Mills, T., Wakeman, M., and Fea, C. (2001). Adult grandchildren's perceptions of emotional closeness and consensus with their maternal and paternal grandparents. J of Family Issues, 22, 427- 432

Moorman Sara (2013) Study Boosts Grandparent-Grandchildren Ties

https://www.bc.edu/bc-web/bcnews/newsarchive/2013/news/study-boostsgrandparent-grandchild-ties.html

Pong, S. L. and Chen V. W. (2010) Coresident Grandparents and Grandchildren's Academic Performance in Taiwan. J Comp Fam Stud. Winter;41(1):111. PMID: 25620815 ; PMCID: PMC4304663.

Sheikh Saleem (2018) Modified Kuppuswamy Scale Updated For Year 2018,Indian J of Research:7 (3)ISSN No. 22501991,217-218.

Shinde, D., and Patnam, V. (2003). Effects of grandparental involvement in bringing 
up grandchildren irrespective of SES. Dissertation submitted to Vasantrao Naik Marathwada Krishi Vidyapeeth, Parbhani.

Sonuga-Barke E J, Mistry M. The effect of extended family living on the mental health of three generations within two
Asian communities. Br J Clin Psychol. 2000 Jun;39(2):129-41. doi: 10.1348/014466500163167. PMID: 10895357.

Wearing, B. (1996). Grand motherhood as leisure? World leisure and recreation 38:4 15-19.

\section{How to cite this article:}

Jaya Bangale and Smita Patil. 2021. The Involvement of Grandparents in Bringing up Grandchildren and their Views About It. Int.J.Curr.Microbiol.App.Sci. 10(12): 391-403. doi: https://doi.org/10.20546/ijcmas.2021.1012.044 